\title{
Prognostic significance of the number of removed lymph nodes according to FIGO stage in ovarian clear cell carcinoma
}

\author{
KOHEI TAMURA, YUJI TAKEI, SHIGEKI MATSUBARA, SUZUYO TAKAHASHI, AKIYO TANEICHI, \\ YOSHIFUMI TAKAHASHI, TAKAHIRO YOSHIBA, TAKAHIRO KOYANAGI, \\ YASUSHI SAGA and HIROYUKI FUJIWARA
}

Department of Obstetrics and Gynecology, Jichi Medical University Hospital, Shimotsuke, Tochigi 329-0498, Japan

Received July 6, 2021; Accepted September 17, 2021

DOI: $10.3892 / \mathrm{mco} .2021 .2425$

\begin{abstract}
A previous study by our group reported that removing a larger number of lymph nodes in patients with stage I ovarian clear cell carcinoma (OCCC) improved progression-free survival (PFS). The present study investigated whether clinical conditions, particularly the number of removed lymph nodes, are independent predictors of progression for stage II or higher OCCC and whether the significance of the number of removed lymph nodes differs according to FIGO stage for OCCC. A total of 113 patients with OCCC who had undergone surgery between January 1993 and December 2015 were retrospectively enrolled and the clinicopathological data were obtained from their medical records. Among patients with stage II or higher OCCC, PFS of those with no residual tumor or no lymph node metastasis was significantly better than that of those with residual tumor $(\mathrm{P}=0.023)$ or lymph node metastasis $(\mathrm{P}=0.035)$. Multivariate analysis revealed that no residual tumor was the only independent predictor for improved PFS of patients with stage II or higher. Regarding the number of removed lymph nodes, it did not significantly affect the PFS of patients with stage II or higher OCCC, whereas it improved the PFS of those with stage I, being an independent predictor of progression of stage I OCCC. In summary, although the number of removed lymph nodes was an independent predictor of progression for stage I OCCC, it was not for stage II or higher OCCC. The prognostic significance of the number of removed lymph nodes in OCCC may differ depending on the FIGO stage.
\end{abstract}

Correspondence to: Dr Yuji Takei, Department of Obstetrics and Gynecology, Jichi Medical University Hospital, 3311-1 Yakushiji, Shimotsuke, Tochigi 329-0498, Japan

E-mail: ytakei@jichi.ac.jp

Abbreviations: OCCC, ovarian clear cell carcinoma; FIGO, Federation of Gynecology and Obstetrics; PFS, progression-free survival; OS, overall survival; ECOG PS, Eastern Cooperative Oncology Group performance status

Key words: clear cell carcinoma, ovarian cancer, progression-free survival, removed lymph nodes, stage

\section{Introduction}

Epithelial ovarian carcinoma generally has poor prognosis; an estimated 313,959 new cases and 207,252 deaths were registered worldwide in 2020 (1). Among epithelial ovarian carcinomas, ovarian clear cell carcinoma (OCCC) demonstrates chemoresistance $(2,3)$ and is more frequent in Japan than in other countries (4). Thus, a novel therapeutic approach for OCCC is required, particularly in Japan.

Although there are several reports on the influence of systematic lymphadenectomy on the prognosis of stage I OCCC, the data are controversial $(5,6)$. A previous study by our group reported that removing more lymph nodes in patients with stage I OCCC increased progression-free survival (PFS), suggesting that the number of removed lymph nodes is an independent predictor of progression for stage I OCCC (7). In that study, the population was confined to patients with stage I OCCC. To the best of our knowledge, there has been no study examining whether the number of removed lymph nodes is an independent predictor of progression for stage II or higher OCCC.

The present article provided a single-institutional retrospective study to investigate whether specific conditions, particularly the number of removed lymph nodes, are independent predictors of progression in patients with stage II or higher OCCC with systematic lymphadenectomy. Furthermore, the significance of the influence of the number of removed lymph nodes was evaluated according to the International Federation of Gynecology and Obstetrics (FIGO) stage of OCCC.

\section{Materials and methods}

Subjects. This study was approved by the ethics committee of Jichi Medical University Hospital (Tochigi, Japan; no. Rindai 15-121). The subjects were patients with stage I to IV OCCC who underwent total hysterectomy, bilateral salpingo-oophorectomy, omentectomy and systematic retroperitoneal lymphadenectomy between January 1993 and December 2015 at Jichi Medical University Hospital (Tochigi, Japan). Patients who had $>20$ lymph nodes removed were included. Exclusion criteria were active double cancer and/or neo-adjuvant chemotherapy. Their medical records were retrospectively reviewed. Those patients with stage I 
up to December 2013 were the same as those in the previous study by our group (7).

Clinical data. Clinicopathological variables, including age, Eastern Cooperative Oncology Group performance status (ECOG PS), complications, tumor diameter, endometriosis, FIGO stage, number of removed lymph nodes, operator, residual tumor, lymph node metastasis, adjuvant chemotherapy, progression, PFS and overall survival (OS) were obtained from the medical records of the subjects. The recurrence site was distinguished between regional node recurrence and others. Cases of simultaneous regional node recurrence and others were assigned to the regional node recurrence group. PFS was defined as the time elapsed between the date of operation and the date of progression or the date of the last follow-up. Progression was defined by the RECIST 1.1 criteria (8). OS was defined as the time elapsed between the date of operation and the date of death or last follow-up. According to internal clinical practice guidelines, the patients were followed up for $>10$ years and follow-up was performed every 1-2 months in the first year, every 2-3 months in the second year, every 3-6 months in the third year, every 4-6 months in the fourth year, every 6 months in the fifth year and yearly thereafter.

Statistical analyses. The best cut-off value of the number of removed lymph nodes for predicting progression was calculated using the receiver operating characteristic curve. The median values were adopted as the cut-off values of age and tumor diameter. The values of age, tumor diameter and number of removed lymph nodes were expressed as median (total range). PFS was estimated using the Kaplan-Meier method. The significance of the difference in survival distribution between subgroups was evaluated using the log-rank test. Variables with $\mathrm{P}<0.05$ in the log-rank test were subsequently entered into the multivariate analysis. Multivariate analysis was performed using Cox's proportional hazards model. $\mathrm{P}<0.05$ was considered to indicate statistical significance. EZR software version 1.54 (Saitama Medical Center, Jichi Medical University) (9) was used for statistical analyses.

\section{Results}

Patients' characteristics. The patient characteristics are presented in Table I. A total of 113 patients were enrolled. The median age was 54 (range, 25-72) years. As for the ECOG PS, 105 patients $(93 \%)$ had an ECOG PS of 0 or 1 and the remaining $8(7 \%)$ had an ECOG PS of 2 or 3 . The number of patients with FIGO stage I, II, III and IV was 77 (68\%), 13 (12\%), 17 (15\%) and $6(5 \%)$, respectively. The main complications were hypertension, hyperlipidemia, diabetes mellites and thrombosis. The others included one case each of a condition such as asthma or hyperthyroidism. The median tumor diameter was 13 (range, 4-28) $\mathrm{cm}$. The median number of removed lymph nodes was 55 (range, 21-135). Endometriosis was diagnosed in 59 patients (52\%). Postoperative adjuvant chemotherapy was performed in 92 patients $(81 \%)$. Progression was noted in 38 patients $(34 \%)$. The calculated cut-off values for the number of removed lymph nodes for predicting progression for stage I and stage II or higher were 33 and 49, respectively.
Table I. Patients' characteristics.

\begin{tabular}{|c|c|}
\hline Characteristic & Value \\
\hline Age, years & $54(25-72)$ \\
\hline \multicolumn{2}{|l|}{ ECOG PS } \\
\hline 0,1 & $105(93)$ \\
\hline 2,3 & $8(7)$ \\
\hline \multicolumn{2}{|l|}{ Stage } \\
\hline I & $77(68)$ \\
\hline II & $13(12)$ \\
\hline III & $17(15)$ \\
\hline IV & $6(5)$ \\
\hline \multicolumn{2}{|l|}{ Complications } \\
\hline HT & $8(7)$ \\
\hline $\mathrm{HL}$ & $7(6)$ \\
\hline DM & $3(3)$ \\
\hline DVT & $3(3)$ \\
\hline $\mathrm{PE}$ & $2(2)$ \\
\hline Others & $32(28)$ \\
\hline Tumor diameter, $\mathrm{cm}$ & $13(4-28)$ \\
\hline Number of removed lymph nodes & $55(21-135)$ \\
\hline \multicolumn{2}{|l|}{ Association with endometriosis } \\
\hline Yes & $59(52)$ \\
\hline No & $54(48)$ \\
\hline \multicolumn{2}{|l|}{ Adjuvant chemotherapy } \\
\hline Yes & $92(81)$ \\
\hline No & $21(19)$ \\
\hline \multicolumn{2}{|l|}{ Progression } \\
\hline Yes & $38(34)$ \\
\hline No & $75(66)$ \\
\hline \multicolumn{2}{|c|}{$\begin{array}{l}\text { Values are expressed as median (range) or n (\%). ECOG PS, Eastern } \\
\text { Cooperative Oncology Group performance status; HT, hypertension; } \\
\text { HL, hyperlipidemia; DM, diabetes mellitus; DVT, deep venous } \\
\text { thrombosis; PE, pulmonary embolism. }\end{array}$} \\
\hline
\end{tabular}

PFS and OS analyses. PFS analyses for patients with stage I are presented in Table II. The PFS of the group with $\geq 33$ removed lymph nodes was significantly better than that of the group with $<33$ removed lymph nodes $(\mathrm{P}=0.001)$. Similarly, the PFS of patients with stage IA was significantly better than that of patients with stage IC $(\mathrm{P}=0.021)$ and that of the group with ECOG PS 0 or 1 was significantly better than that of the group with ECOG PS 2 or $3(\mathrm{P}<0.001)$. The PFS curves according to the number of removed lymph nodes, stage and ECOG PS are presented in Fig. 1. OS analyses for patients with stage I are presented in Table II. Only ECOG PS 0 or 1 was a good prognostic factor for $\mathrm{OS}(\mathrm{P}=0.005)$.

PFS analyses for patients with stage II or higher are presented in Table III. The PFS of patients with no residual tumor or no lymph node metastasis was significantly better than that of those with residual tumor $(\mathrm{P}=0.023)$ or lymph node metastasis $(\mathrm{P}=0.035)$. However, there were no significant differences in PFS based on the number of removed lymph 
Table II. Log-rank test in the survival analysis for stage I patients based on the Kaplan-Meier method.

\begin{tabular}{|c|c|c|c|c|c|}
\hline Variable & $\begin{array}{c}\text { Number of } \\
\text { patients }\end{array}$ & $\begin{array}{c}\text { 5-year } \\
\text { PFS (\%) }\end{array}$ & P-value & $\begin{array}{l}\text { 5-year } \\
\text { OS }(\%)\end{array}$ & P-value \\
\hline Age, years & & & 0.223 & & 0.891 \\
\hline$<54$ & 40 & 70 & & 82 & \\
\hline$\geq 54$ & 37 & 86 & & 88 & \\
\hline ECOG PS & & & $<0.001$ & & 0.005 \\
\hline 0,1 & 73 & 80 & & 87 & \\
\hline 2,3 & 4 & 25 & & 33 & \\
\hline Complication & & & 0.855 & & 0.782 \\
\hline Yes & 40 & 77 & & 86 & \\
\hline No & 37 & 78 & & 83 & \\
\hline Tumor diameter, cm & & & 0.657 & & 0.710 \\
\hline$<13$ & 33 & 79 & & 81 & \\
\hline$\geq 13$ & 44 & 77 & & 88 & \\
\hline Association with endometriosis & & & 0.306 & & 0.226 \\
\hline Yes & 47 & 72 & & 80 & \\
\hline No & 30 & 87 & & 93 & \\
\hline Stage & & & 0.021 & & 0.079 \\
\hline IA & 21 & 100 & & 100 & \\
\hline $\mathrm{IC}$ & 56 & 69 & & 79 & \\
\hline Number of removed lymph nodes & & & 0.001 & & 0.148 \\
\hline$\geq 33$ & 65 & 83 & & 87 & \\
\hline$<33$ & 12 & 46 & & 70 & \\
\hline Operator & & & 0.220 & & 0.202 \\
\hline Gynecological oncologist & 60 & 75 & & 82 & \\
\hline Other & 17 & 88 & & 94 & \\
\hline Adjuvant chemotherapy & & & 0.669 & & 0.647 \\
\hline Yes & 58 & 76 & & 86 & \\
\hline No & 19 & 83 & & 83 & \\
\hline
\end{tabular}

PFS, progression-free survival; OS, overall survival; ECOG PS, Eastern Cooperative Oncology Group performance status.
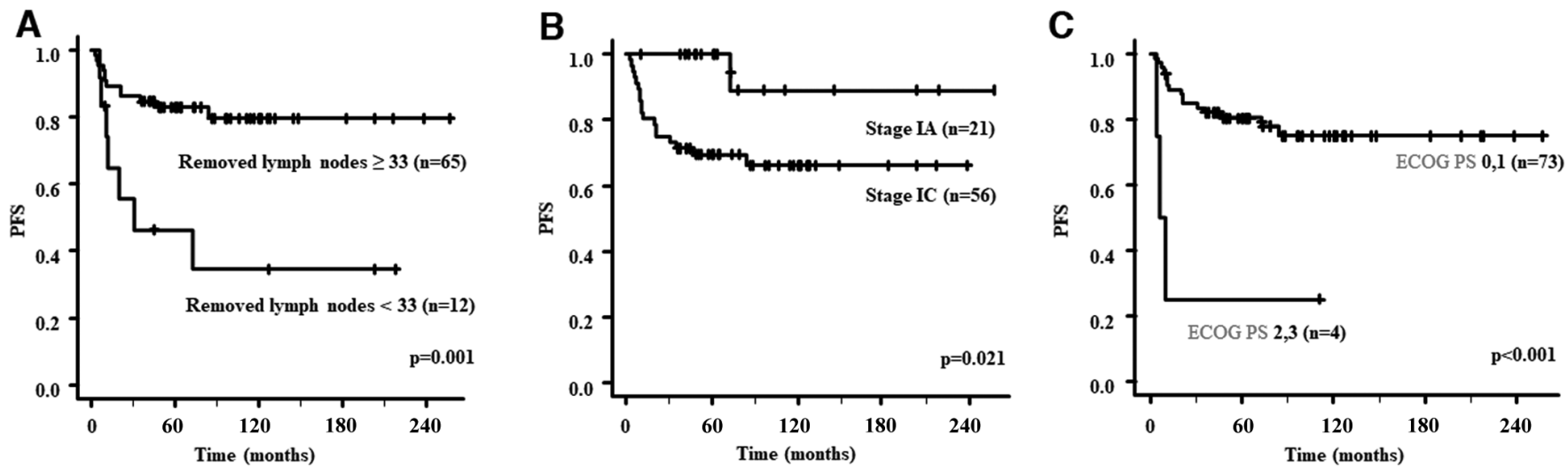

Figure 1. Kaplan-Meier curves of PFS for stage I patients. (A) Number of removed lymph nodes $\geq 33$ vs. the number of removed lymph nodes $<33$. (B) Stage IA vs. stage IC. (C) ECOG PS 0 or 1 vs. ECOG PS 2 or 3. PFS, progression-free survival; ECOG PS, Eastern Cooperative Oncology Group performance status.

nodes. PFS curves according to residual tumor and lymph node metastasis are provided in Fig. 2. OS analyses for patients with stage II or higher are presented in Table II. There was no significant prognostic factor for OS. 
Table III. Log-rank test in the survival analysis for patients with stage II or higher based on the Kaplan-Meier method.

\begin{tabular}{|c|c|c|c|c|c|}
\hline Variable & $\begin{array}{c}\text { Number of } \\
\text { patients }\end{array}$ & $\begin{array}{c}\text { 5-year } \\
\text { PFS (\%) }\end{array}$ & P-value & $\begin{array}{l}\text { 5-year } \\
\text { OS }(\%)\end{array}$ & P-value \\
\hline Age, years & & & 0.641 & & 0.745 \\
\hline$<54$ & 16 & 50 & & 59 & \\
\hline$\geq 54$ & 20 & 50 & & 70 & \\
\hline ECOG PS & & & 0.586 & & 0.455 \\
\hline 0,1 & 32 & 50 & & 64 & \\
\hline 2,3 & 4 & 75 & & 75 & \\
\hline Complication & & & 0.116 & & 0.537 \\
\hline Yes & 15 & 40 & & 66 & \\
\hline No & 21 & 59 & & 66 & \\
\hline Tumor diameter, $\mathrm{cm}$ & & & 0.834 & & 0.594 \\
\hline$<13$ & 20 & 50 & & 65 & \\
\hline$\geq 13$ & 16 & 56 & & 64 & \\
\hline Association with endometriosis & & & 0.282 & & 0.225 \\
\hline Yes & 12 & 58 & & 75 & \\
\hline No & 24 & 47 & & 60 & \\
\hline Stage & & & 0.082 & & 0.233 \\
\hline II & 13 & 69 & & 77 & \\
\hline III, IV & 23 & 40 & & 59 & \\
\hline Number of removed lymph nodes & & & 0.468 & & 0.090 \\
\hline$\geq 49$ & 21 & 56 & & 76 & \\
\hline$<49$ & 15 & 47 & & 50 & \\
\hline Lymph node metastasis & & & 0.035 & & 0.083 \\
\hline Yes & 12 & 31 & & 50 & \\
\hline No & 24 & 63 & & 74 & \\
\hline Operator & & & 0.111 & & 0.417 \\
\hline Gynecological oncologist & 29 & 53 & & 68 & \\
\hline Other & 7 & 43 & & 57 & \\
\hline Residual tumor & & & 0.023 & & 0.100 \\
\hline Yes & 12 & 17 & & 44 & \\
\hline No & 24 & 66 & & 75 & \\
\hline
\end{tabular}

PFS, progression-free survival; OS, overall survival; ECOG PS, Eastern Cooperative Oncology Group performance status.
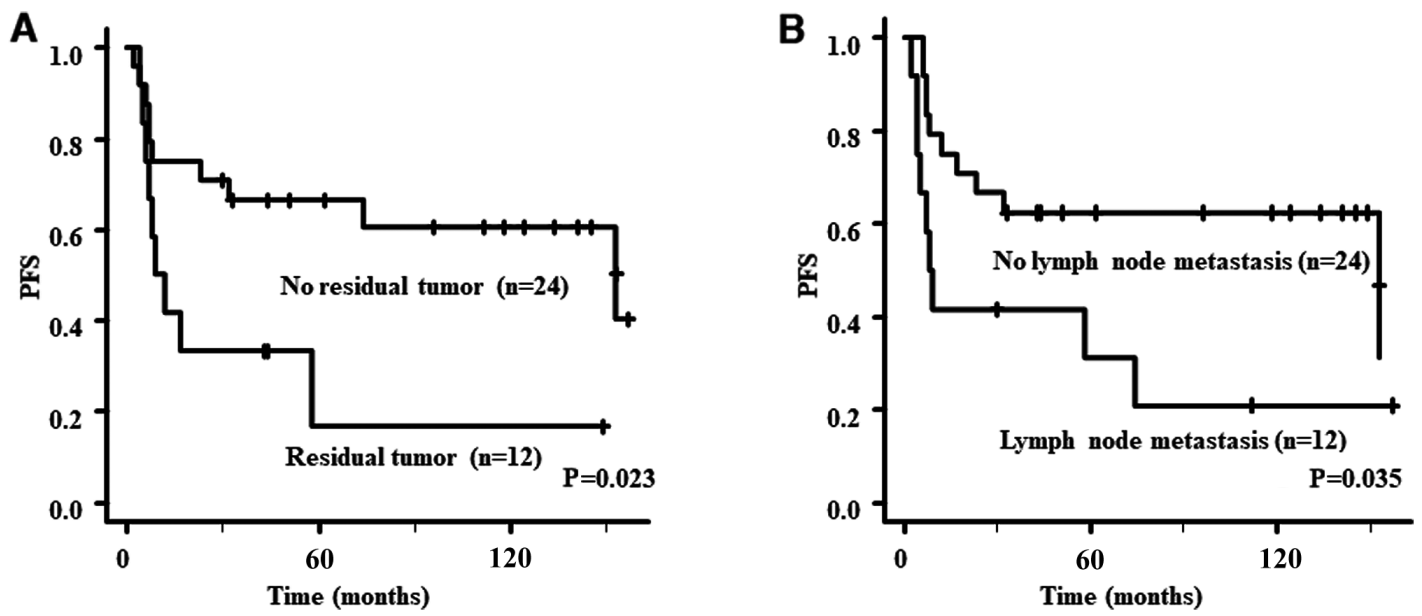

Figure 2. Kaplan-Meier curves of PFS for stage II or higher patients. (A) No residual tumor vs. residual tumor. (B) No lymph node metastasis vs. lymph node metastasis. PFS, progression-free survival. 
Table IV. Multivariate analysis for predictors of progression for stage I.

\begin{tabular}{lccc}
\hline Variable & $\begin{array}{c}\text { Number of } \\
\text { patients }\end{array}$ & $\begin{array}{c}\text { Hazard ratio } \\
(95 \% \mathrm{CI})\end{array}$ & P-value \\
\hline $\begin{array}{l}\text { ECOG PS } \\
0,1\end{array}$ & 73 & 1 & $<0.001$ \\
2,3 & 4 & $14.3(3.27-62.4)$ & \\
Stage & & & 0.026 \\
IA & 21 & 1 & \\
IC & 56 & $10.4(1.32-82.6)$ & \\
Number of removed & & & 0.004 \\
lymph nodes & & & \\
$\geq 33$ & 65 & 1 & \\
$<33$ & 12 & $4.06(1.55-10.6)$ & \\
\hline
\end{tabular}

95\% CI, 95\% confidence interval; ECOG PS, Eastern Cooperative Oncology Group performance status.

Multivariate analyses for predictors of progression. Multivariate analyses were performed to identify independent predictors of progression. For patients with stage I, the number of removed lymph nodes, stage and ECOG PS were compared. The results are presented in Table IV. A number of removed lymph nodes of $\geq 33$, stage IA and ECOG PS 0 or 1 were independent predictors of improved PFS ( $\mathrm{P}=0.004,0.026$ and $<0.001$, respectively).

For patients with stage II or higher, residual tumor and lymph node metastasis were assessed as predictors of PFS. The results are presented in Table V. No residual tumor was the only independent predictor of improved PFS $(\mathrm{P}=0.048)$.

Recurrence sites. Regarding stage I, the site of recurrence was consistent with the previous study by our group (7), as an additional 9 cases did not recur and there was no significant difference (data not shown). According to stage II-IV, the recurrence site was clearly identified in 18 patients. Of 12 patients with $\geq 49$ removed lymph nodes, 3 (25\%) had regional node recurrence. Recurrence sites in the other 9 patients (75\%) were peritoneal dissemination, pleural dissemination, lung, liver, thymus and inguinal node. On the other hand, of the 6 patients with $<49$ removed lymph nodes, 2 (33\%) had regional node recurrence. Recurrence sites of the other 4 patients $(67 \%)$ were peritoneal dissemination, the diaphragm, liver and spleen. There was no significant difference in the incidence of regional node recurrence between the group with $\geq 49$ removed lymph nodes and that with $<49$.

\section{Discussion}

In the present study, it was examined whether the number of removed lymph nodes is an independent predictor of progression in patients with stage II or higher OCCC with systematic lymphadenectomy and the significance of the number of removed lymph nodes according to the FIGO stage In OCCC was investigated. The results revealed that, in stage II or higher
Table V. Multivariate analysis for predictors of progression for stage II or higher.

\begin{tabular}{lccc}
\hline Variable & $\begin{array}{c}\text { Number of } \\
\text { patients }\end{array}$ & $\begin{array}{c}\text { Hazard ratio } \\
(95 \% \text { CI })\end{array}$ & P-value \\
\hline $\begin{array}{l}\text { Lymph node } \\
\text { metastasis }\end{array}$ & & & 0.070 \\
$\quad$ No & 24 & 1 & \\
$\quad$ Yes & 12 & $2.32(0.93-5.78)$ & \\
Residual tumor & & 1 & 0.048 \\
$\quad$ No & 24 & $2.62(1.01-6.80)$ & \\
Yes & 12 & & \\
\hline
\end{tabular}

95\% CI, 95\% confidence interval.

OCCC, although residual tumor is an independent predictor of progression, the number of removed lymph nodes is not. This is contradictory to the results on stage I OCCC, as previously reported by our group (7): The number of removed lymph nodes was an independent predictor of progression, which was reconfirmed by the present study including added data of stage I OCCC.

The first novel finding of the present study was that the number of removed lymph nodes is not a predictor of progression for stage II or higher OCCC. There have been numerous reports on the therapeutic significance of systematic lymphadenectomy for ovarian cancer. A recent randomized trial (LION trial) demonstrated that systematic lymphadenectomy in patients with advanced ovarian cancer who underwent intraabdominal macroscopical complete resection and had normal lymph nodes is not associated with a longer OS or PFS than with no lymphadenectomy (10). However, the majority of patients in the LION trial had serous carcinoma and patients with OCCC comprised only $2.2 \%$ (10). As OCCC is chemoresistant, unlike serous carcinoma and endometrioid carcinoma, it must be considered separately from other histological types. There are several reports on the therapeutic significance of systematic lymphadenectomy for OCCC. Systematic lymphadenectomy was reported to both improve $(11,12)$ and not improve the prognosis $(6,13)$. Therefore, its therapeutic significance for OCCC is controversial.

Focusing on the number of removed lymph nodes, to the best of our knowledge, there are only two reports in which the prognosis was investigated based on the number of removed lymph nodes for OCCC $(5,7)$, one of which is the previous study by our group. Regarding the number of removed lymph nodes, $>20$ was selected to avoid including resection of only swollen lymph nodes. Similarly, a recent study defined systematic lymphadenectomy as at least 20 removed lymph nodes (14). The present study added 9 patients with stage I OCCC. Patients were enrolled during the 2 years after the previous study by our group (7), providing a 13\% increase in the patient number, and all patients with stage I OCCC who met the criteria were reanalyzed. This analysis reconfirmed the previous results that the number of removed lymph nodes is an independent predictor of progression for stage I OCCC. The two previous reports described stage I OCCC $(5,7)$. Therefore, the present 
study was the first to examine the impact of the number of resected lymph nodes on stage II or higher OCCC. According to the present data and clinical experience at our clinic, in contrast to stage I, tumors are likely to spread throughout the body in stage II or higher, particularly in stages III and IV. Even if there are no macroscopic tumors after surgery, microscopic tumors may remain. As OCCC is chemoresistant, OCCC patients with microscopic tumors may have a high risk of recurrence. Even if more lymph nodes are removed, it may not be possible to prevent recurrence in patients with stage II or higher OCCC with microscopic tumors.

The second finding was that residual tumor is an independent predictor of progression for stage II or higher OCCC. This is consistent with the previous report demonstrating that only cytoreductive surgery resulting in no residual tumor is able to improve the prognosis of advanced OCCC (15). Patients with no residual tumor had significantly better PFS than those with a tumor $<1 \mathrm{~cm}(\mathrm{P}=0.04)$ or those with a tumor $>1 \mathrm{~cm}$ $(\mathrm{P}<0.01)(15)$. This is reasonable considering that OCCC is chemoresistant.

Since clinical stage I ovarian CCC had about 4.5-7.5\% lymph node metastasis $(5,6,16)$, the clinical stage I ovarian CCC cases may have included pathological stage III cases at about 4.5-7.5\%. Therefore, removing more lymph nodes leads to accurate staging and an improved prognosis for stage I OCCC. On the other hand, in stage II or higher OCCC, complete surgery without macroscopic residual tumor may be important regardless of the number of removed lymph nodes and lymph node metastasis. Although speculative, the gynecologic surgeon may only remove the swollen lymph nodes instead of systematic lymphadenectomy in stage II or higher OCCC.

A limitation of present study was that the study population was small and that this may have been a source of low significance of the results. In future studies, larger cohorts from multiple centers should be investigated. In particular, as the number of patients was small, it was difficult to perform subgroup analyses. Initially, it was intended to analyze stage II OCCC in the same manner as stage I OCCC, as clinical stage II OCCC may include pathological stage III cases. If more lymph nodes are removed in patients with clinical stage II OCCC, a certain proportion of them may have been diagnosed with pathological stage III. Therefore, the number of removed lymph nodes may also be an independent predictor of progression for stage II OCCC. However, as there were only 13 cases of stage II OCCC, it was not possible to analyze stage II. It was also considered that the number of patients was insufficient for OS analysis. Since all patients of the present study were subjected to systematic lymphadenectomy, it was not possible to make a comparison between the group with systematic lymphadenectomy and that with non-systematic lymphadenectomy. Therefore, how systematic lymphadenectomy affects survival in OCCC compared with non-systematic lymphadenectomy will be the focus of future studies.

In conclusion, the present study revealed that the number of removed lymph nodes is not a predictor of progression for stage II or higher OCCC, differing from stage I OCCC. Residual tumor was an independent predictor of progression for stage II or higher OCCC. Although further studies with a large number of patients are required to confirm the results, the present study provides useful information on how to perform retroperitoneal lymphadenectomy according to the FIGO stage for OCCC.

\section{Acknowledgements}

Not applicable.

\section{Funding}

No funding was received.

\section{Availability of data and materials}

The datasets analyzed during the current study are available from the corresponding author upon reasonable request.

\section{Authors' contributions}

KT, SM, HF and YT designed the present study, critically revised the manuscript and analyzed data. ST, AT, YT, TY, TK and YS performed the data collection. KT wrote the manuscript. KT and YT checked and confirmed the authenticity of the raw data. All authors read and approved the final manuscript.

\section{Ethics approval and consent to participate}

This study was approved by the ethics committee of Jichi Medical University Hospital (Tochigi, Japan; no. Rindai 15-121). Patient consent was not required, as this study was a retrospective observational study, but the patients or their families were given the opportunity to opt out.

\section{Patient consent for publication}

Not applicable.

\section{Competing interests}

The authors declare that they have no competing interests.

\section{References}

1. Sung H, Ferlay J, Siegel RL, Laversanne M, Soerjomataram I, Jemal A and Bray F: Global Cancer Statistics 2020: GLOBOCAN estimates of incidence and mortality worldwide for 36 cancers in 185 countries. CA Cancer J Clin 71: 209-249, 2021.

2. Takei Y, Suzuki M, Ohwada M, Saga Y, Kohno T, Machida S and Sato I: A feasibility study of paclitaxel and carboplatin therapy in Japanese patients with epithelial ovarian cancer. Oncol Rep 10: 951-955, 2003.

3. Sugiyama T, Kamura T, Kigawa J, Terakawa N, Kikuchi Y, Kita T, Suzuki M, Sato I and Taguchi K: Clinical characteristics of clear cell carcinoma of the ovary: A distinct histologic type with poor prognosis and resistance to platinum-based chemotherapy. Cancer 88: 2584-2589, 2000.

4. Machida H, Matsuo K, Yamagami W, Ebina Y, Kobayashi Y, Tabata T, Kanauchi M, Nagase S, Enomoto T and Mikami M: Trends and characteristics of epithelial ovarian cancer in Japan between 2002 and 2015: A JSGO-JSOG joint study. Gynecol Oncol 153: 589-596, 2019.

5. Mahdi H, Moslemi-Kebria M, Levinson KL, Gojayev A, Lockhart D, Ali-Fehmi R and Munkarah AR: Prevalence and prognostic impact of lymphadenectomy and lymph node metastasis in clinically early-stage ovarian clear cell carcinoma. Int J Gynecol Cancer 23: 1226-1230, 2013. 
6. Takano M, Sugiyama T, Yaegashi N, Suzuki M, Tsuda H, Sagae S, Udagawa Y, Kuzuya K, Kigawa J, Takeuchi S, et al: The impact of complete surgical staging upon survival in early-stage ovarian clear cell carcinoma: A multi-institutional retrospective study. Int J Gynecol Cancer 19: 1353-1357, 2009.

7. Takei Y, Takahashi S, Machida S, Taneichi A, Yoshiba T, Takahashi Y, Yoshida C, Saga Y, Matsubara S and Fujiwara H: Impact of the number of removed lymph nodes on recurrence-free survival in stage I ovarian clear cell carcinoma. Int J Clin Oncol 23: 930-935, 2018.

8. Eisenhauer EA, Therasse P, Bogaerts J, Schwartz LH, Sargen D, Ford R, Dancey J, Arbuck S, Gwyther S, Mooney M, et al: New response evaluation criteria in solid tumours: Revised RECIST guideline (version 1.1). Eur J Cancer 45: 228-247, 2009.

9. Kanda Y: Investigation of the freely available easy-to-use software 'EZR' for medical statistics. Bone Marrow Transplant 48: 452-458, 2013.

10. Harter P, Sehouli J, Lorusso D, Reuss A, Vergote I, Marth C, Kim JW, Raspagliesi F, Lampe B, Aletti G, et al: A randomized trial of lymphadenectomy in patients with advanced ovarian neoplasms. N Engl J Med 380: 822-832, 2019.

11. Yamazaki H, Todo Y, Shimada C, Takeshita S, Minobe S, Okamoto K, Yamashiro K and Kato H: Therapeutic significance of full lymphadenectomy in early-stage ovarian clear cell carcinoma. J Gynecol Oncol 29: e19, 2018.
12. Magazzino F, Katsaros D, Ottaiano A, Gadducci A, Pisano C, Sorio R, Rabaiotti E, Scambia G, Cormio G, Scarampi L, et al: Surgical and medical treatment of clear cell ovarian cancer: Results from the multicenter Italian Trials in Ovarian Cancer (MITO) 9 retrospective study. Int J Gynecol Cancer 21: 1063-1070, 2011.

13. Kajiyama H, Suzuki S, Yoshikawa N, Tamauchi S, Shibata K and Kikkawa F: The impact of systematic retroperitoneal lymphadenectomy on long-term oncologic outcome of women with advanced ovarian clear-cell carcinoma. J Gynecol Oncol 31: e47, 2020.

14. Nasioudis D, Latif NA, Haggerty AF, Giuntoli Ii RL, Kim SH and Ko EM: Outcomes of comprehensive lymphadenectomy for patients with advanced stage ovarian carcinoma and rare histologic sub-types. Int J Gynecol Cancer 31: 1132-1136, 2021.

15. Takano M, Kikuchi Y, Yaegashi N, Kuzuya K, Ueki M, Tsuda H, Suzuki M, Kigawa J, Takeuchi S, Tsuda H, et al: Clear cell carcinoma of the ovary: A retrospective multicentre experience of 254 patients with complete surgical staging. Br J Cancer 94: 1369-1374, 2006

16. Mueller JJ, Holzapfel M, Han CH, Santos K, Gunderson C, Moore K, Erickson B, Leath CA III, Diaz E, Walsh C, et al: Staging lymphadenectomy in patients with clear cell carcinoma of the ovary. Int J Gynecol Cancer 26: 120-124, 2016. 